\title{
Search for extra spatial dimensions at DØ
}

\author{
Lisa Berntzon* ${ }^{\dagger}$ \\ CPPM Marseille, France \\ E-mail: berntzon@cppm.in2p3.fr
}

\section{Introduction}

In this talk results from searches for Large Extra spatial Dimensions (LEDs) and RandallSundrum (RS) gravitons at $\mathrm{D} \varnothing$ are presented, detailed information about the searches presented in this talk can be found in $[1,2,3,4]$. The results from the search for $\mathrm{TeV}^{-1}$-size extra dimensions can be found in [5]. Large Extra spatial Dimensions (LEDs) was proposed by Arkani-Hamed, Dimopoulos and Dvali (ADD) [6], they postulate that the standard model (SM) particles and gauge interactions are confined to a three-dimensional "brane" in a bulk which consists of 3 plus $n$ additional compact spatial dimensions. This removes the hierarchy problem as gravity appears weak, $\left(M_{E W}<<M_{P l}\right)$, because it propagates in the bulk. The reduced Planck scale $M_{D}$ is the fundamental scale in the $(4+n)$ space-time. To be able to 'dilute' gravity in the volume of the extra dimensions, so that it appears weak in the 3D-world, these compact extra dimensions need to be 'large'. The size of the LEDs, the compactification radius $R$, is given by: $R^{n}=M_{P l}^{2} /\left(8 \pi M_{D}^{n+2}\right)$. Gravitons propagate in the bulk and they appear as a tower of Kaluza-Klein (KK) excited modes from the point of view of the SM-brane and can be detected via virtual graviton exchange or direct graviton emission.

International Europhysics Conference on High Energy Physics

July 21st - 27th 2005

Lisboa, Portugal

\footnotetext{
${ }^{*}$ Speaker.

† for the DØ Collaboration

${ }^{\ddagger}$ Partially fi nanced by Stockholm University.
} 
Lisa Randall and Raman Sundrum has in [7] proposed a rigorous solution of the hierarchy problem, by adding a single extra dimension with a non-Euclidian, warped metric (RS-model). The size of the ED is $R$ and the curvature of the warped metric is $k$, (the warp factor). The hierarchy problem is solved by localization of gravity to the Planck brane, at $\phi=0$, while the SM fi elds are localised on the TeV-brane at $\phi=\pi$, where $\phi$ is the coordinate along the ED. Gravity originates on the Planck brane and the graviton wave function is exponentially suppressed away from the brane along the ED due to the warp-factor. The $M_{P l}$-size operators on the Planck brane yield low-energy effects on the SM brane with a typical scale of $\Lambda_{\pi}=\bar{M}_{P l} \exp (-k \pi R)$, where $\bar{M}_{P l} \equiv M_{P l} / \sqrt{8 \pi}$ is the reduced Planck mass. The hierarchy problem is solved if $\Lambda_{\pi} \sim 1 \mathrm{TeV}$, which can be achieved by requiring $k R \sim 10$. In the simplest RS model only gravitons propagate in the bulk, and can be detected as low mass resonances, the lowest order of these KK-modes is massless, and the searches presented here thus put a limit on $M_{1}$, the mass of the fi rst resonance. The parameters $k$ and $R$ can be expressed in $M_{1}$ and the coupling to the SM fi elds: $k \bar{M}_{P l}$. The theoretically prefered range of $M_{1}$ is a few hundred $\mathrm{GeV}$ to a few $\mathrm{TeV}$, while $k / \bar{M}_{P l}$ is expected to be between $0.01-0.1$.

\section{Searches for LEDs via virtual graviton exchange.}

Virtual graviton exchange can be detected as an enhancement at high masses of the di-lepton or di-photon mass spectrum. The change of the cross section due to the interference of gravity effects is: $d^{2} \sigma / d M d \cos \theta^{*}=f_{s m}+f_{\text {int }} \eta_{G}+f_{K K} \eta_{G}^{2}$, where $f_{\text {sm }}$ is the SM-term, $f_{\text {int }}$ is the interference and $f_{K K}$ is the graviton terms, $M$ is the invariant di-lepton or di-photon mass, and $\theta^{*}$ is the scattering angle. The effects of LEDs are parametrised by a single variable $\eta_{G}=F / M_{s}^{4}$. The dimensionless parameter $F$ of order unity reflects the dependence of the cross section on the different formalisms for the effective Langrangian: GRW [8], HLZ [9] and Hewett [10]. The only difference between the formalisms is the defi nitions of $M_{s}$, which is the ultra-violet cut-off required to keep the divergent sum over KK states fi nite. Virtual graviton effects are sensitive only to $M_{s}$ and not $M_{D}$, but the two scales are expected to be of the same order. $M_{s}$ could be slightly lower, in which case virtual graviton searches are more sensitive to LEDs than direct graviton searches. The analyses presented here are performed in the dielectron, diphoton and dimuon channels [1,2]. To maximise the reconstruction effi ciency for electrons and photons, the tracking information was ignored and these channels were combined in a single calorimeter based 'diEM' channel. The EM-objects were required to be isolated, to have tranverse energy $E_{T}>25 \mathrm{GeV}$ and to be in central or forward regions of the calorimeter, but with at least one of the 2 objects in the central region. Events with more than 2 high- $\mathrm{p}_{T}$ EM objects were rejected. The muons were identifi ed in the muon spectrometer and were required to have a matching track in the central tracking detector, transverse momentum $p_{T}>15 \mathrm{GeV}$, be isolated and pass a cosmic veto. The main backgrounds are Drell-Yan (DY) and direct $\gamma \gamma$ production, and instrumental background from jets misidentifi ed as EM objects ('QCD' background). The former was estimated using simulation and the QCD background was estimated from data. The resulting invariant mass spectra are shown in Fig. 1 and Fig. 2, the data is in agreement with the expected background. Limits on $M_{s}$ are set using the distributions of $M$ and $\cos \theta^{*}$ in a 2D likelihood fit of the parameter $\eta_{G}$. In the diEM channel, using $200 \mathrm{pb}^{-1}$ of data, a new $95 \%$ C.L limit of $M_{s}$ in the GRW convention of $1.36 \mathrm{TeV}$ is set and combined with the RunI result the limit is $1.43 \mathrm{TeV}$. In the dimuon channel, using $246 \mathrm{pb}^{-1}$ of data, 95\% C.L limits of $M_{s}$ between 
0.85 and $1.27 \mathrm{TeV}$ are set in the different formalisms. These are the most stringent limits on LEDs to date.

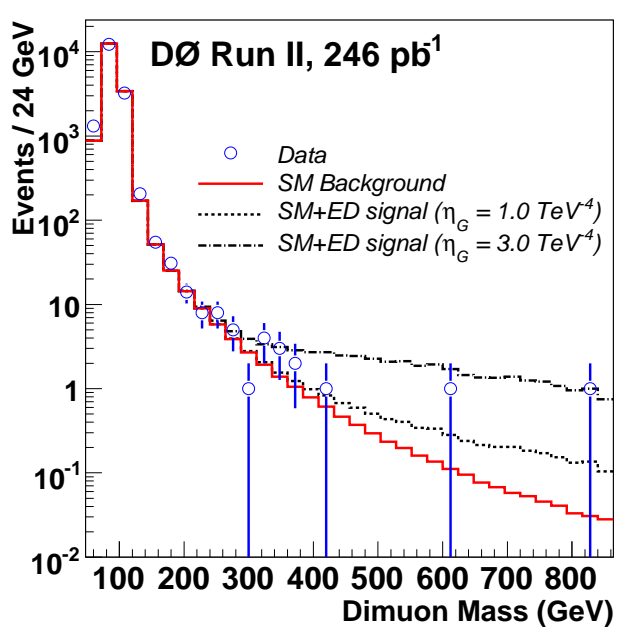

Figure 1: The dimuon mass distribution for data and SM background in the LED search, the effects of LED are shown for $\eta_{G}=1 \mathrm{TeV}^{-4}$ and $3 \mathrm{TeV}^{-4}$ in dashed lines.

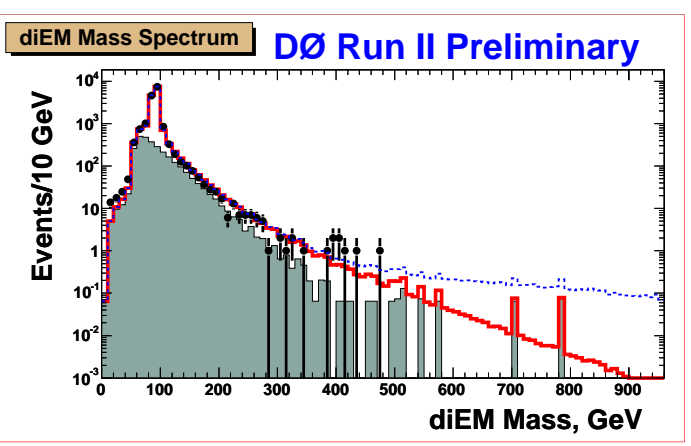

Figure 2: The diEM Mass distribution for data (errorbars), QCD background (histogram), and a fit to the SM background and QCD (full line). The dashed line show the effect of the ED signal for $\eta_{G}=0.6$ $\mathrm{TeV}^{-4}$.

\section{Searches for LEDs via direct graviton emission.}

At the Tevatron gravitons can be produced recoiling against a quark or a gluon jet, which results in a monojet-like topology. The analysis presented here is the fi rst preliminary result from Run II of searches for LEDs in the monojet channel [3]. The search is performed using events with single jets and acoplanar jets with missing $E_{T}$. Data was collected using a trigger specially designed for Jets $+\mathbb{E}_{T}$ topologies. The leading jet $E_{T}$ and $\mathbb{E}_{T}$ were required to exceed $150 \mathrm{GeV}$, no additional jet with $E_{T}>50 \mathrm{GeV}$ was allowed, and the angle (jet, $\mathbb{E}_{T}$ ) was required to be at least $30^{\circ}$. The resulting missing $E_{T}$ spectra is shown in Figure 3, as well as the uncertainty due to the jet energy scale (JES). The challenge in this analysis is the large instrumental background from $\mathbb{E}_{T}$-mismeasurements and the sensitivity to uncertainty of the JES. Nevertheless, using only $85 \mathrm{pb}^{-1}$ the $95 \%$ C.L. limit on $M_{D}$ is $0.68 \mathrm{TeV}$ for $n=4$, which is better than the DØ Run I limit. This analysis is being updated using more data and improved JES.

\section{Direct search for Randall-Sundrum gravitons.}

In the RS-model, the excited KK-modes can decay into fermion-antifermion or diboson pairs and be detected as a resonance in the invariant mass spectrum. The analysis presented here is a direct search for RS-gravitons in the dielectron, diphoton and dimuon fi nal states [4]. As for the LED-search, the electron and photon channel was combined into a 'diEM' channel to maximise the reconstruction effi ciency. The requirements on the muons and EM-objects as well as the main 


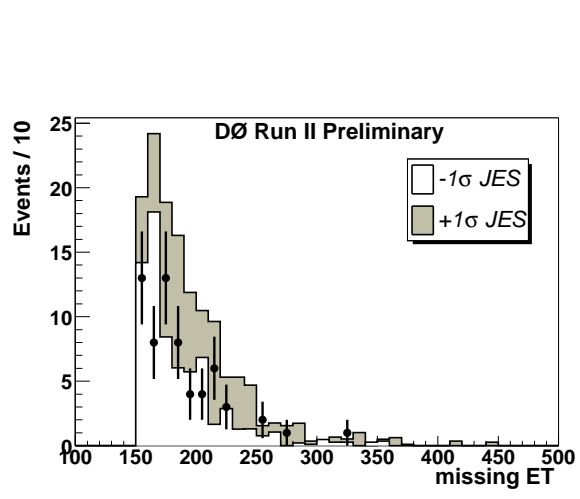

Figure 3: The $\mathbb{E}_{T}$ distribution of the jets $+\mathbb{E}_{T}$ channel, the uncertainty due to JES is also shown.
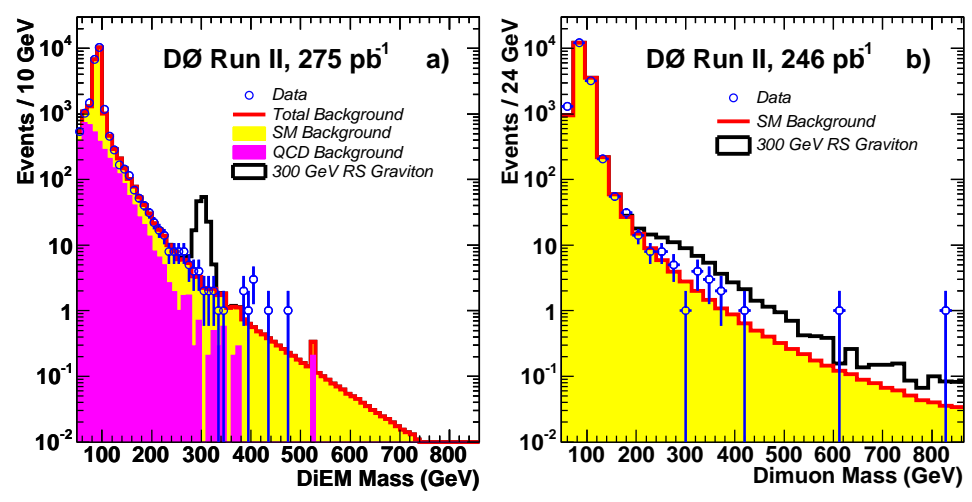

Figure 4: The resulting invariant mass spectra of the RS-graviton search in the diEM channel, (to the right) and the dimuon channel (to the left).

backgrounds are similar to the ones described in Section 2. The resulting invariant mass spectra are shown in Figure 4, the data is in agreement with the expected background, and limits on $M_{1}$ are set using the mass window method. A combination of the diEM-channel using $275 \mathrm{pb}^{-1}$ of data, and the dimuon channel, using $246 \mathrm{pb}^{-1}$, excludes graviton masses up to $785(250) \mathrm{GeV}$, for $k / M_{P l}=$ 0.1 (0.01), at 95\% C.L.. These are the most stringent limits on RS-gravitons to date.

\section{References}

[1] DØ Collaboration, Search for Large Extra Spatial Dimensions in Dimuon Production at DØ, Phys. Rev. Lett. 95161602 (2005), FERMILAB-PUB-05/250-E, [hep-ex/ 0506063 ].

[2] DØ Collaboration, Search for Large Extra Dimensions in the Dielectron and Diphoton Channels with $200 \mathrm{pb}^{-1}$ of Run II Data, DØNote 4336-CONF.

[3] DØ Collaboration, Search for large extra spatial dimensions in Jets + Missing $E_{T}$ topologies, DØNote 4400-CONF.

[4] DØ Collaboration, Search for Randall-Sundrum Gravitons in Dilepton and Diphoton Final States, Phys. Rev. Lett. 95091801 (2005), FERMILAB-PUB-05/126-E, [hep-ex/0 505018 ].

[5] DØ Collaboration, Search for Large and $\mathrm{TeV}^{-1}$ Extra Dimensions in the Dielectron channel with 200 $\mathrm{pb}^{-1}$ of Data, DØNote 4349-CONF.

[6] N. Arkani-Hamed, S. Dimopoulos and G. Dvali, Phys. Lett. B429, 263 (1998), Phys. Rev. D. 59, 086004 (1999); I. Antoniadis, N. Arkani-Hamed, S. Dimopoulos and G. Dvali, Phys. Lett. B436, 257 (1998).

[7] L. Randall, R. Sundrum, Phys. Rev. Lett. 833370 (1999), Phys. Rev. Lett. 834690 (1999).

[8] G. Giudice, R. Rattazzi, and J. Wells, Nucl. Phys. B544, 3 (1999) [hep-ph/9811291 ].

[9] T. Han, J. D. Lykken and R. -J. Zhang, [hep-ph/9811350 ].

[10] J. L. Hewett, Phys. Rev. Lett. 82, 4765 (1999). 